\title{
15. \\ Exemplum usus functionum iteratarum in theoria functionum integraliter transcendentium.
}

(Auct. Hill, prof. math. Lond. Goth.)

Functiones iteratae maximum habent usum in unacunque fere analyseos parte, praecipue in summando aequationes ad differentias, nec minorem in integrando aequationibus differentialibus. In problemate vero quadrandi et ad multipla transcendentium invenienda et ad comparationem rite instuendam maximi conducunt. Cujus rei in praesenti tantummodo exempla proponamus aliqua. Primum vero exemplum idoneae substitutionis exhibeamus.

Sit functio $g x$ ejusmodi, ut $g(g x)$ seu $g^{2} x=x$ sit, atque ponatur $\int g x d x=G x$, eritque universim

$$
G x+G(g x)=x g x+\text { Const. }
$$

Est enim $d\left(x_{g} x\right)=d x \cdot g x+x d(g x)$, atque $d G(g x)=d g \dot{x} \cdot G_{1}(g x)=$ $d g x \cdot g(g x)$ seu $d G(g x)=x \cdot d g x$, ideoque $d(x g x)=d G x+d G(g x)$, unde integrando formula proposita sponte fluit. Sit ex. gr. grx $=\sqrt{ }\left(a^{n}-x^{n}\right)$, quo casu $(g x)^{n}=a^{n}-x^{n}, g^{2} x=g(g x)={ }^{n}\left(a^{n}-(g x)^{n}\right)$ seu $g^{2} x={ }^{n} x^{n}=x$. Si itaque statiumus $G x=\int_{0}^{x} d x \sqrt{n}^{n}\left(a^{n}-x^{n}\right),(G 0=0)$, erit

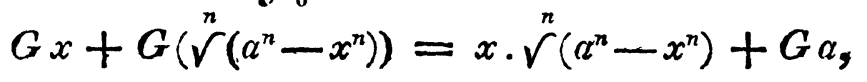

speciatimque, si $x=\stackrel{n}{v}^{n}\left(a^{n}-x^{n}\right)=\frac{a}{\sqrt[n]{ } 2}=c, 2 G c=c^{2}+G a$. Sin vero ponis $n=-r$, formulam habebis similem, specialem quidem, pro functione $\int \frac{x \partial x}{v^{v}\left(x^{r}-a^{r}\right)}$ comparanda. Has speciales formulas quis facile immediate conjiciat, attamen generalis nostra formula latissime patet. Sit enim $\boldsymbol{\gamma} \boldsymbol{x}$, functio quaecunque ipsius $x$, atque $\bar{\gamma} x$ ipsius inversa, ponaturque $g x=$ $\bar{\gamma}(-\gamma x)$, eritque $g x$ indolis ab initio desideratae, nempe $g^{2} x=g x$. Est enim $g^{2} x=g(g \cdot x)=\bar{\gamma}(-\gamma g x)=\bar{\gamma}(\gamma x)=x$, quoniam $\gamma(g \cdot x)=\gamma \bar{\gamma}(-\gamma x)$ $=-\gamma x$. Si igitur $\gamma x$ neque par neque impar fuerit functio, innumerae dabunfur functiones $\xi x$, atque transcendentes $G x\left(={ }_{0}^{x} g x d x\right)$ correspon- 
dentes, quarum aliqua saltem proprietas innotuerit: haec nempe $G x+G g x=x \cdot g x+G g 0$.

At ulterius progrediamur.

Si nempe similiter fuerit functio $g$ ejusmodi ut $g^{3} x=x$ sit, ponaturque $G x=\int_{0}^{x}\left(d x \cdot g x \cdot g^{2} x\right)$, erit

$$
G x+G g x+G\left(g^{2} x\right)=x \cdot g x \cdot g^{2} x+\text { Const. }
$$

Quoniam enim $d G x=d x \cdot g x \cdot g^{2} x$, erit substituendo $g x$ in locum ipsius $x$, $d G_{g} x=d g x \cdot g g^{g} x \cdot g^{2} g x=d g x \cdot g^{2} x \cdot x$, (ob $\left.g^{2}(g x)=g^{3} x=x\right)$, iterumque $d G g^{2} x=d g g x \cdot g^{2} g x \cdot g x=d g^{2} x \cdot x \cdot g x$; ideoque

$d G x+d G g x+d G g^{2} x=d x \cdot g x \cdot g^{2} x \cdot x+d g x \cdot g^{g^{2}} x \cdot x+d g^{2} x \cdot x \cdot g x$, quod aperte $=d\left(x \cdot g x \cdot g^{2} x\right)$ aequatur, unde integrando formula modo proposita consequitur. Constante vero rite definito erit

$$
G x+G g x+G g^{2} x=x g x g^{2} x+G g 0+G g^{2} 0 .
$$

Iam vero accipiendo $c=$ radici ex aequatione $x=g x$, erit $g c=c$, atque $g^{2} c=g g c=g c=c$, ideoque

$$
G c+G g c+G g^{2} c=3 G c=c \cdot g c \cdot g^{2} c+G g 0+G g^{2} 0,
$$

seu $3 G c=c^{3}+G g 0+G g^{2} 0$. Sin vero $g 0=0$ fuerit, erit $G c=\frac{\pi}{3} c^{3}$ $\operatorname{seu}^{c} \int_{0} d x \cdot g x \cdot g^{2} x=\frac{x}{3} c^{3}$, quod integrale definitum est satis memorabile, cum ad innumeras functionum formas quadret.

Iam vero patet, universim fore

$G x+G g x+G\left(g^{2} x\right)+\ldots .+G\left(g^{r-1} x\right)=x \cdot g x \cdot g^{g^{2}} x \ldots g^{g-1} x+$ Const., si fuerit $g^{r} x=x$ atque $G x=\int_{0}^{x} d x \cdot g x \cdot g^{2} x \ldots . g^{r-1} x$; eritque Const. $=0$, si $g 0=0$, alias vero

$$
\text { Const. }=G g 0+G g^{2} 0+\ldots . .+G_{g^{r-1}} 0 .
$$

Huius vero formulae demonstrandi ars ex antecedentibus facile oolligitur. Speciatem vero, si fuerit $c=g c$, similiter colligitur, fore $r G c=c^{r}+$ Const., ideoque $\int_{0}^{c} d x \cdot g x \cdot g^{2} x \ldots . g^{r-1} x=\frac{1}{r} c^{r}$, si $g 0=0$ fuerit; alias vero, si altera datur radix $c^{\prime}$ aequationis $x=g x$, erit similiter $r G c^{\prime}=c^{\prime r}+$ Const., ideoque $r\left(G c^{\prime}-G c\right)=c^{r r}-c^{r}$, seu ${ }^{c} \int_{c} d x \cdot g x \cdot g^{g^{2}} x \ldots . g^{r-1} x=\frac{c^{r}-c^{r}}{r}$, quod est integrale definitum maxime momenti, utut et latissime patens, (cum $g x$ arbitraria fere sit functio), et haudquaquam antiquorum indolis, cum horum pleraque, ni valde fallimur, ad extremum eo nituntur, ut functio differentialis nihilo vel infinito aequetur. Rei vero amplitudinem ita comprobamus. Sit $\gamma x$ functio quaecunque (coefficientibus imaginariis 
praedita, si placet), ejusque inversa $\bar{\gamma} x$ (i. e. si aequatio $\gamma x=y$ dissolvitur, praebeat $x=\bar{\gamma} y$, seu $x=\gamma^{(-1)} y$, exsistente -1 iterationis exponente), ponaturque ad interim $g x=\bar{\gamma} e \gamma x$, eritque $g(g x)=\bar{\gamma} e \gamma g x$, seu $g^{2} x=\bar{\gamma} e^{2} \gamma x$, cum $\gamma g x=e \gamma x$ sit; similiter erit ulterius $g^{2}(g x)=$ $\bar{\gamma} e^{2}\left(\gamma_{g}^{g} x\right)$, seu $g^{3} x=\bar{\gamma} e^{3} \gamma x$, generatimque igitur $g^{r} x=\bar{\gamma} e^{r} \gamma x$. Ut vero jam $g^{r r} x=x$ sit, ponatur $e^{r}=1$, quo facto erit $\delta^{r} x=\bar{\gamma} \gamma x=x$. Si igitur accipiamus $e=v^{r} 1=$ radici imaginarii ex unitate, et $\gamma$ et $g$, ut modo diximus, erit equidem $g^{r} x=x$, ideoque $g x$ et $G x\left(=\int_{0}^{x} d x g x g^{2} x \ldots g^{r-1} x\right)$ indolis modo explicatae. Patet vero, partim coefficientes functionis $y x$ ita (imaginaria forsan) determinari posse, ut $g x$ realis evadit, partim $G x$ semper realem esse, cum ejus differentiale symmetrica sit functio radicis solius imaginariae ${ }^{r}$.

Nemo vero non videt, ex universalibus modo exhibitis formulis innumera consequi tum integralia definita tum integralia comparandi regulas. Tantum igitur exempli causa unum alterumve exponamus.

Sit videlicet $g x={ }^{n}\left(a+b x^{n}\right)$, eritque $g^{r} x=V^{n}\left(a \cdot \frac{1-b^{r}}{1-b}+b^{r} x\right)$, quandoquidem

$$
g^{r+1} x=g^{r} x=\sqrt{n}\left(a \cdot \frac{1-b^{r}}{1-b}+b^{r}\left(a+b x^{n}\right)\right)=V^{n}\left(a \cdot \frac{1-b^{r+1}}{1-b}+b^{r+1} x^{n}\right) .
$$

Iam vero accipiatur $b^{m}=1$, nec $b=1$, eritque $g^{m} x=\sqrt{ }^{n} x^{n}=x$, ideoque, ponendo $d G x=d x \cdot g x \cdot g^{\sigma^{2}} x \ldots \cdot g^{m-1} x$, ex modo dictis

$G x+G g x+G g^{g^{2}} x+\ldots+G g^{m-1} x=x g x \cdot g^{g^{2}} x \ldots g^{m-1} x+$ Const. Iam vero, si fuerit $c=g c=\mathfrak{n}^{n}\left(a+b c^{n}\right)$, seu $c=\sqrt[n]{ }\left(\frac{a}{1-b}\right)$, aut etiam $a=(1-b) c^{n}$, erit

$\int_{0}^{c} d x V\left[\left[\left(a+b x^{n}\right)\left(a(1+b)+b^{2} x^{n}\right)\left(a\left(1+b+b^{2}\right)+b^{3} x^{n}\right) \ldots .\left(a \cdot \frac{1-b^{r}}{1-b}+b^{r} x^{n}\right)\right]=\frac{1}{m}\left(\frac{a}{1-b}\right)^{\frac{m}{n}}+\right.$ Cons1.

seu $G c=\frac{1}{m}\left(c^{m}+\right.$ Const. $)$, ubi $r=m-1$, atque Const. $=G g \cdot 0+G g^{2} 0+\ldots+G g^{r} 0$.

Hoc vero integrale definitum satis abstrusum (nam $n$ valorem quemvis admittit), quamquam speciale, tamen latissime patet. Primum vero observamus, si capiatur $\frac{a}{1-b}=\alpha=$ reale, id quod sub radicis signo est, cum symmetrica ipsorum $b$ sit functio, fore reale; sin vero ponatur $a=$ $(1-b) c^{n}$, erit factoris cujusvis forma haec:

$$
c^{n}\left(1-b^{r}\right)+b^{r} x \text { seu } c^{n}+b^{r}\left(x^{n}-c^{n}\right) \text {. }
$$


Ex. gr. si ponamus $n=2, r=2, m=3$, erit integrale nostrum

$$
\int d x v\left[\left(c^{2}+b\left(x^{2}-c^{2}\right)\right)\left(c^{2}+b^{2}\left(x^{2}-c^{2}\right)\right)\right]=\int d x r\left(3 c^{4}-3 c^{2} x^{2}+x^{4}\right) \text {. }
$$

Sin vero ponatur $n=-2$, atque $m=3$, erit

$$
\int \frac{c^{2} x^{2} d x}{\sqrt{\left(c^{4}-3 c^{2} x^{2}+3 x^{4}\right)}}
$$

Si igitur $x=c z$, rel, quod eodem redit, $c=1$, et erit

$$
\int_{0}^{z} d z \sqrt{ }\left(3-3 z^{2}+z^{4}\right)=E z \text {, atque } \int_{0}^{z} \frac{z^{2} d z}{\sqrt{\left(1-3 z^{2}+3 z^{4}\right)}}=G z \text {, }
$$

speciatimque

$$
\begin{aligned}
& E z+E g z+E g^{2} z=z \cdot g z \cdot g^{2} z+E g 0+E g_{g}^{2} 0, \\
& G z+G g z+G g^{2} z=z \cdot g z \cdot g^{2} z+G g 0+G g^{2} 0,
\end{aligned}
$$

atque

$$
\begin{aligned}
& 3 . E 1=1+E g 0+E g^{2} 0 \\
& 3 . G 1=1+G g 0+G g^{2} 0,
\end{aligned}
$$

ubi $g \theta=r(1-b), g^{2} 0=r\left(1-b^{2}\right)$, existente $b=v^{3} 1=\frac{-1 \pm V(-3)}{2}$. Calculanti vero mox patebit, integralia definita $E 1$ et $G 1$ ad ipsa attinere Elliptica, quae cel. Legendre in Exc. d. c. int. T. 1. p. 54. pertractavit. Patet vero alteram radicem aequationis $z=g z\left[=\sqrt{ }\left(1+b\left(z^{2}-1\right)\right)\right]$, fore -1, ideoque

$$
\int_{-1}^{+1} \frac{z^{2} d z}{\sqrt{ }\left(3 z^{4}-3 z^{2}+1\right)}=\frac{1}{3} \text {, atque } \int_{-1}^{+1} d z r\left(3-3 z^{2}+z^{4}\right)=\frac{1}{3} \text {. }
$$

1 Si vero posueris $n=2, m=4$, vel $=5$ etc., casum habebis in functionibus Abelianis aeque memorabilem, ac istum in Ellipticis.

Ulterius ponendo $B x=\mathbb{V}^{n}\left(\frac{a+a_{1} x^{n}}{b+b_{1} x^{n}}\right)$, certamque relationem inter coefficientes $a, a_{1}, b$ et $b_{1}$ (exsistente exponente $n$ arbitrario), habelis $\operatorname{g}^{r} x=x$, indeque theoremata similia de functione

$$
\int d x V^{n} /\left[\left(\frac{a+a_{1} x^{n}}{b+b_{1}^{0} x^{n}}\right) \cdot\left(\frac{a^{2}+a_{1}^{2} x^{n}}{b^{2}+b_{1}^{1} x^{n}}\right) \ldots\left(\frac{a^{r}+a_{1}^{r} x^{n}}{b^{r}+b_{1}^{r-1} x^{n}}\right)\right]
$$

ubi ibidem casus $r=2$ ellipticas, $r$ vero $=3,4$ etc. Abelianas tangit functiones. Haec vero indicasse sufficiat, cum particularia in praesenti minus curemus *).

*) Indicasse igitur sufficiat, alia obtineri integralia definita, ponendo $c=\delta_{i}^{2} c$, vel $c=g^{2} c$ etc. Ex. gr. $c=g^{2} c$, et $g^{m} x=x$, erit $g^{3} c=\delta c, g^{4} c=g^{2} c=c$, generatimque $g^{2 r} c=c$ atque $g^{2 r+1} c=g c$; ideoque $n(G c+G g c)=(c g c)^{n}+$, Const., si $m=2 n$. 
Nemo vero non videt, hanc theoriam pleniorem functionum iteratarum cognitionem postulare; quare et completam de his doctrinam jam octo abhinc annis composuimus, quam et jam nobis publicis faciendi juris est animus, dummodo editor idoneus sumtus suppeditat.

Londini Gothorum d. V. ante cal. Jul. 1833.

Sin vero $m=2 n+1$ impar fuerit, erit $(n+1) G c+n G_{g} c=c^{n+1}(g c)^{n}+$ Const.; ubi Const. idem ac antea.

In exemplis allatis nonnisi speciales functionum comparandarum regulas ohtinuimus. Attamen rem hac via accuratius adgrediendo generales quidem comparandi formulas obtinuimus, idque ita, ut non modo, quidquid hucusque in arte quadrandi calluerimus, hinc quasi ex proprio fonte explicetur, sed etiam nơva regulae investigentur.

Hinc enim vel integrale indefinitum, seu inter limites arbitrarias $x$ et $y$ sumtum, aequatione quadam resolvendo definimus, vel geverales ipsum comparandi formulas elicimus. Prioris rei, comprobandae causa heic absque explicatione appouimus formulas :

$$
\begin{aligned}
& \text { 1. } \int_{x} \frac{d x}{f^{10} x}=r \text {, si } y=f^{r} x \text { fuerit; } \\
& \text { 2. } \int_{u}^{x} \frac{{\frac{p^{\prime}}{}} x d x}{\left(f^{\prime 0} x\right)^{2}}=\frac{\varphi^{r} x}{f^{\prime 0} u} \text {, si } u=f^{r} x \text {; }
\end{aligned}
$$

quae theoremata aeque memorabilia constituunt, de quilus aliquando posthac agemus. 